\title{
SYNTHESIS AND ANTI-MICROBIAL ACTIVITIES OF 1,3,5-TRISUBSTITUTED-PYRAZOLE DERIVATIVES CONTAINING A PYRIDYL MOIETY
}

\author{
DUN-JIA WANG*, HUA LIU, YAN-FANG KANG, YAN-JUN HU, XIAN-HONG WEI \\ Hubei Collaborative Innovation Center for Rare Metal Chemistry, College of Chemistry and Chemical Engineering, \\ Hubei Normal University, Huangshi 435002, China
}

\begin{abstract}
Claisen condensation of ethyl isonicotinate with different acetophenones gave the corresponding pyridyl- $\beta$-diketones, while the treatment with hydrazine hydrate yielded 3,5-disubstituted-1 $H$-pyrazoles, which then converted 1,3,5-trisubstituted-pyrazole derivatives containing a pyridyl moiety by $\mathrm{N}$-acylation with acyl chloride. The structures of all newly synthesized compounds are established by FTIR, ${ }^{1} \mathrm{H}$ NMR, mass spectroscopy and elemental analysis, and in the case of compound 2e, analyzed by single-crystal X-ray diffraction further. The anti-microbial activities of the title compounds have been tested by disc diffusion method against Escherichia coli, Staphylococcus aureus, Pyricularia oryzae and Rhizoctnia solani. The results showed that compounds $\mathbf{3 c}$ and $\mathbf{4 c}$ exhibited good inhibitory activity against all the tested organisms.
\end{abstract}

Key words: $1,3,5$-Trisubstituted-pyrazole, $\beta$-Diketone, Synthesis, Anti-microbial activity

\section{INTRODUCTION}

Pyrazole derivatives are an interesting class of organic compounds, which are found to be associated with diverse pharmacological properties such as antimicrobial [1,2], anti-inflammatory [3], antihypertensive [4], antidepressant [5], antiviral [6] and anticancer [7] activities. The synthesis and applications of these compounds have received considerable attention in recent years [8-10]. In addition, the pyridyl ring is also a prominent heterocyclic scaffold in lot of bioactive molecules. Many pyridine-based compounds have been reported to exhibit versatile bioactivities, such as insecticidal, fungicidal, anticancer and antibacterial activities [11-13]. Keeping this in view, we choose the pyridyl ring as one of the aryl substituents about the central pyrazole ring to synthesize a series of new 1,3,5-trisubstituted-pyrazole derivatives containing a pyridyl moiety. Herein we synthesized some novel 1-acyl-3-(4-pyridyl)-5aryl-pyrazole derivatives and presented the initial results of anti-microbial activities against Escherichia coli, Staphylococcus aureus, Pyricularia oryzae and Rhizoctnia solani of the title compounds.

\section{RESULTS AND DISCUSSION}

\subsection{Chemistry}

The synthesis of 1,3,5-trisubstituted-pyrazole derivatives is conducted as outlined in Scheme 1. The pyridyl- $\beta$-diketones 1a-e were prepared via Claisen condensation of the required methyl ketones with ethyl isonicotinate in benzene using sodium amide as the condensing agent. The structures of 1a-e were established under the basis of their elemental analysis and spectral data. Their ${ }^{1} \mathrm{H}$ NMR spectra exhibited the single peaks at $\delta=4.60-4.68$ attributed to the keto- $\mathrm{CH}_{2}$ protons, the single peaks at $\delta=6.83-6.93$ assigned to the vinylic protons in enol and the single peaks at $\delta=16.52-16.68$ due to the enolic $\mathrm{OH}$ protons. The IR spectra data also demonstrated the presence of the $\mathrm{C}=\mathrm{O}$ and enolic $\mathrm{C}=\mathrm{C}$ stretching vibrations at $1600-1591 \mathrm{~cm}^{-1}$ and $1545-1511 \mathrm{~cm}^{-1}$ in the pyridyl- $\beta$-diketones, respectively.

Treatment of the pyridyl-b-diketones 1a-e with hydrazine monohydrate in refluxing ethanol for $3 \mathrm{~h}$ gave the $1 H$-pyrazoles $2 \mathrm{a}$-e. The IR spectra for compounds 2a-e displayed the typical bands of the N-H stretching vibrations at $3135-3115 \mathrm{~cm}^{-1}$ and their ${ }^{1} \mathrm{H}$ NMR spectra exhibited the single peaks at $\delta=$ 6.85-7.00 due to the 4-position protons of $1 H$-pyrazoles, but the N-H resonance of the pyrazole ring were not observed in $\mathrm{CDCl}_{3}$. This is in agreement with the result in the literature [14]. In the case of $\mathbf{2 e}$, the structure was additionally solved by single-crystal X-ray diffraction. There are two molecules (A and $\mathbf{B})$ in the asymmetric unit in the crystal structure. The labeled displacement ellipsoid plots of these two molecules are shown in Fig. 1. The comparison between molecule (A) and molecule (B) shows no significant differences in bond lengths and bond angles. However, their torsion angles and dihedral angles between the phenyl ring and pyrazole ring exhibit some differences in the molecules. The torsion angles $\mathrm{C} 6-\mathrm{C} 1-\mathrm{C} 7-\mathrm{C} 8, \mathrm{C} 2-\mathrm{C} 1-\mathrm{C} 7-\mathrm{C} 8, \mathrm{C} 6-\mathrm{C} 1-\mathrm{C} 7-\mathrm{C} 12, \mathrm{C} 2-\mathrm{C} 1-\mathrm{C} 7-\mathrm{C} 12$, $\mathrm{C} 11-\mathrm{C} 10-\mathrm{C} 15-\mathrm{C} 13$ and $\mathrm{C} 9-\mathrm{C} 10-\mathrm{C} 15-\mathrm{C} 13$ are $153.16(0.19)^{\circ},-25.36(0.29)^{\circ}$, $-26.02(0.28)^{\circ}, 155.47(0.21)^{\circ},-27.05(0.31)^{\circ}$ and $150.25(0.21)^{\circ}$ in molecule (A), respectively, while C21-C26-C27-C28, C25-C26-C27-C28, C21-C26$\mathrm{C} 27-\mathrm{C} 32, \mathrm{C} 25-\mathrm{C} 26-\mathrm{C} 27-\mathrm{C} 32, \mathrm{C} 31-\mathrm{C} 30-\mathrm{C} 33-\mathrm{C} 34$ and $\mathrm{C} 29-\mathrm{C} 30-\mathrm{C} 33-\mathrm{C} 34$ are $147.95(0.21)^{\circ},-31.02(0.29)^{\circ},-31.47(0.29)^{\circ}, 149.56(0.20)^{\circ},-20.95(0.31)^{\circ}$ and $158.28(0.19)^{\circ}$ in molecule $(\mathbf{B})$, respectively. The dihedral angles for the two benzene rings and the benzene ring with the pyrazole ring are $25.86^{\circ}$ and $25.97^{\circ}$ in molecule (A), respectively, but in molecule (B), the dihedral angles are $31.30^{\circ}$ and $21.65^{\circ}$, respectively.

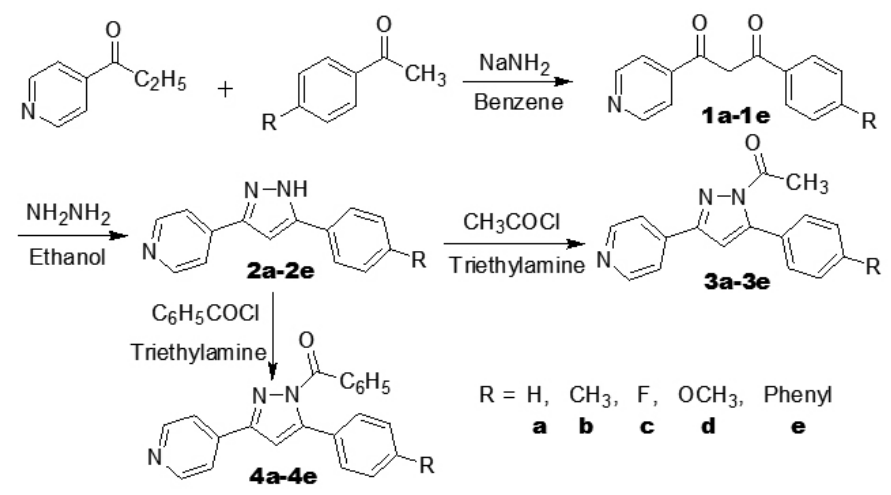

Scheme 1. The synthetic routes for compounds 3a-e and 4a-e.

The title compounds 3a-e and 4a-e were obtained by N-acylation of the $1 H$-pyrazoles 2a-e with acyl chloride in the presence of triethylamine. Their structures were confirmed by elemental analysis, FTIR, ${ }^{1} \mathrm{H}$ NMR and mass spectroscopy. Their IR spectra showed the absorption bands at $1740-1710 \mathrm{~cm}^{-1}$ attributed to the $\mathrm{C}=\mathrm{O}$ stretching vibration of the $\mathrm{N}$-acylation products. Their ${ }^{1} \mathrm{H}$ NMR spectra revealed the single peaks at $\delta=6.73-7.19$ corresponding to the vinylic protons of the pyrazole ring. The molecular ion peaks $\left(\mathrm{M}^{+}\right)$for these compounds were observed in accordance with the Nitrogen Rule. The mass spectra of the title compounds were compared to confirm elemental compositions.

\subsection{Anti-microbial activity}

The screening results of in vitro anti-microbial activities of compounds 3a-e and 4a-e are summarized in Table 1. All test data were the average values from triplicate runs. It is obvious from the data that these compounds possess 
inhibitory activities to a certain degree against the tested microorganisms. It was observed that compounds $\mathbf{3 c}$ and $\mathbf{4 c}$ exhibited much higher activities against Escherichia coli, Staphylococcus aureus, Pyricularia oryzae and R. solani among all synthesized compounds, but compounds $\mathbf{3 d}$ and $\mathbf{4 d}$ revealed much lower activities. By the preliminary structure-activity relationship analysis, it was concluded that compounds 3a-e had slightly better anti-microbial activities than compounds $\mathbf{4 a - e}$ attributed to the strong electron-withdrawing property of the $\mathrm{N} 1$ acetyl group in the pyrazole nucleus [15]. Especially, the pyrazole derivatives with 4-fluorophenyl moiety at 5-position of the pyrazole ring showed potent anti-microbial activities against the tested microorganisms, being significantly higher than the rest $(\mathrm{R}=$ phenyl, 4-methylphenyl, 4-methoxyphenyl and 4-phenylphenyl). As a result, it indicated that the electronic nature of the substituent groups at para positions in the phenyl ring played a significant role in anti-microbial activities.

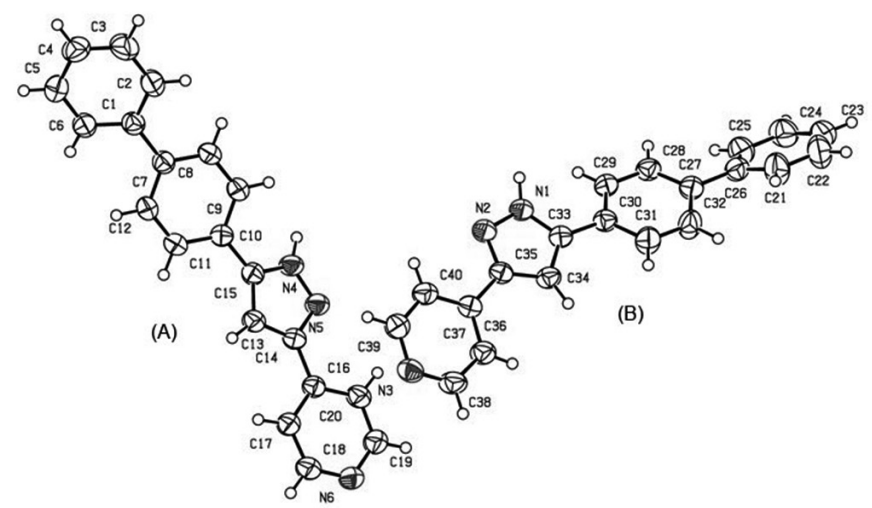

Fig. 1 View of compound $2 \mathrm{e}$ with the atom-labeling scheme and $50 \%$ probability displacement ellipsoids.

Table 1 Anti-microbial data of 1,3,5-trisubstituted-pyrazole derivatives.

\begin{tabular}{|c|c|c|c|c|}
\hline \multirow[t]{2}{*}{ Compound } & \multicolumn{2}{|c|}{$\begin{array}{c}\text { Antibacterial activity } \\
\text { Zone of Inhibition in } \\
\mathrm{mm}\end{array}$} & \multicolumn{2}{|c|}{$\begin{array}{c}\text { Antifungal activity Zone } \\
\text { of Inhibition in mm }\end{array}$} \\
\hline & E. coli & S. aureus & P. oryzae & R. solani \\
\hline $3 a$ & 14 & 15 & 16 & 13 \\
\hline $3 b$ & 11 & 12 & 12 & 10 \\
\hline $3 c$ & 22 & 21 & 23 & 22 \\
\hline $3 d$ & 9 & 10 & 12 & 11 \\
\hline $3 e$ & 16 & 16 & 15 & 16 \\
\hline $4 a$ & 13 & 15 & 14 & 12 \\
\hline $4 b$ & 11 & 9 & 10 & 12 \\
\hline $4 c$ & 20 & 21 & 22 & 20 \\
\hline $4 d$ & 10 & 9 & 10 & 8 \\
\hline $4 e$ & 17 & 15 & 16 & 15 \\
\hline Norfloxacin & 31 & 30 & - & - \\
\hline Triadimefon & - & - & 28 & 30 \\
\hline
\end{tabular}

\section{CONCLUSIONS}

In conclusion, a series of new 1,3,5-trisubstituted-pyrazole derivatives containing a pyridyl moiety were synthesized by Claisen condensation, cyclization and $\mathrm{N}$-acylation and their structures were characterized by FTIR, ${ }^{1} \mathrm{H}$ NMR, MS spectroscopy and elemental analysis. The anti-microbial activities of these compounds were evaluated against Escherichia coli, Staphylococcus aureus, Pyricularia oryzae and Rhizoctnia solani. The results showed that these compounds showed certain degree of anti-bacterial and anti-fungal activities and compounds $\mathbf{3} \mathbf{c}$ and $\mathbf{4 c}$ were much higher active against the organisms employed among all synthesized compounds. Thus, this study provides a lead for synthesis and evaluation of more pyrazole derivatives for anti-microbial activity as the same could lead to the discovery of some potential agents.

\section{EXPERIMENTAL}

Melting points were determined using X-4 digital melting point apparatus and are uncorrected. Infrared spectra were recorded on a Nicolet FTIR 5700 spectrophotometer with $\mathrm{KBr}$ pellets. The ${ }^{1} \mathrm{H}$ NMR spectra were measured on an advance $\mathrm{III}^{\mathrm{TM}} 300 \mathrm{MHz}$ NB Digital NMR spectrometer in $\mathrm{CDCl}_{\text {, solution with }}$ TMS as internal standard. Electrospray ionization mass spectra (ESI-MS) were performed with a Finnigan LCQ Advantage Max spectrometer. The elemental analysis $(\mathrm{C}, \mathrm{H}, \mathrm{N})$ was carried out on a Perkin-Elmer 2400 elemental analyzer. Reagents were of analytical grade and were used without further purification.

4.1 Typical procedure for the synthesis of the pyridyl- $\beta$-diketones

To a suspension of sodium amide $(1.4 \mathrm{~g}, 36 \mathrm{mmol})$ and ethyl isonicotinate $(6.65 \mathrm{~g}, 44 \mathrm{mmol})$ in benzene $(50 \mathrm{~mL})$, A solution of the aryl methyl ketones $(22 \mathrm{mmol})$ in benzene was added dropwise under stirring at $50{ }^{\circ} \mathrm{C}$. The reaction mixture was refluxed for about $7 \mathrm{~h}$ until the dark yellow product precipitated. The precipitate was filtered off and washed with $5 \%$ acetic acid until $\mathrm{pH}=6$. The crude products were recrystallized from ethanol $(95 \%)$ to give the pyridyl$\beta$-diketones (1a-e).

4.1.1 1-Phenyl-3-(4-pyridyl)-1,3-propandione (1a)

Colorless needles, yield $70.5 \%, \mathrm{mp} 81-82^{\circ} \mathrm{C} ;{ }^{1} \mathrm{H}$ NMR $\left(\mathrm{CDCl}_{3}, 300 \mathrm{MHz}\right)$ $\delta: 4.65\left(\mathrm{~s}, 0.08 \mathrm{H}\right.$, keto $\left.\mathrm{CH}_{2}\right), 6.89(\mathrm{~s}, 1 \mathrm{H}$, enol CH$), 7.50-7.63(\mathrm{~m}, \mathrm{Ar}-\mathrm{H}, 3 \mathrm{H})$, $7.79(\mathrm{~d}, 2 \mathrm{H}, \mathrm{Py}-\mathrm{H}, J=5.7 \mathrm{~Hz}), 8.01(\mathrm{~d}, 2 \mathrm{H}, \mathrm{Ar}-\mathrm{H}, J=7.8 \mathrm{~Hz}), 8.80(\mathrm{~d}, 2 \mathrm{H}$ Py-H, $J=5.7 \mathrm{~Hz}$ ), 16.55 (br s, 1H, enol OH) ppm; IR (KBr) n: 3419 (w), 3089 $(\mathrm{m}), 3040(\mathrm{~m}), 2920(\mathrm{~m}), 1591(\mathrm{~s}), 1542(\mathrm{~s}), 1480(\mathrm{~m}), 1401(\mathrm{~m}), 1290(\mathrm{~m})$, $1228(\mathrm{~m}), 1064$ (m), 764 (s), $692(\mathrm{~s}) \mathrm{cm}^{-1}$; ESI-MS m/z: $226.13[\mathrm{M}+1]^{+}$. Anal. calcd. for $\mathrm{C}_{14} \mathrm{H}_{11} \mathrm{NO}_{2}$ : C 74.65, H 4.92, N 6.22; found C 74.89, H 4.88, N 6.25.

4.1.2 1-(4-Methylphenyl)-3-(4-pyridyl)-1,3-propandione (1b)

Yellow crystals, yield $68.2 \%, \mathrm{mp} 102-103{ }^{\circ} \mathrm{C}$; ${ }^{1} \mathrm{H}$ NMR $\left(\mathrm{CDCl}_{3}, 300\right.$ $\mathrm{MHz}) \mathrm{d}$ : 2.44 (s, 3H, $\left.\mathrm{CH}_{3}\right), 4.62$ (s, $0.02 \mathrm{H}$ keto $\left.\mathrm{CH}_{2}\right), 6.86(\mathrm{~s}, 1 \mathrm{H}$, enol $\mathrm{CH})$, $7.31(\mathrm{~d}, 2 \mathrm{H}, \mathrm{Ar}-\mathrm{H}, J=8.1 \mathrm{~Hz}), 7.78(\mathrm{~d}, 2 \mathrm{H}, \mathrm{Py}-\mathrm{H}, J=5.7 \mathrm{~Hz}), 7.91(\mathrm{~d}, 2 \mathrm{H}$, Ar-H, $J=8.1 \mathrm{~Hz}), 8.79(\mathrm{~d}, 2 \mathrm{H}, \mathrm{Py}-\mathrm{H}, J=5.7 \mathrm{~Hz}), 16.61(\mathrm{br} \mathrm{s}, 1 \mathrm{H}$, enol OH) ppm; IR (KBr) n: 3434 (w), 3108 (m), 3029 (m), 2919 (m), 1599 (s), 1534 (s), $1486(\mathrm{~s}), 1296(\mathrm{~m}), 1231(\mathrm{~m}), 1056(\mathrm{~m}), 982(\mathrm{~m}), 839(\mathrm{~m}), 792(\mathrm{~s}) \mathrm{cm}^{-1}$; ESIMS m/z: $240.05[\mathrm{M}+1]^{+}$. Anal. calcd. for $\mathrm{C}_{15} \mathrm{H}_{13} \mathrm{NO}_{2}: \mathrm{C} 75.30, \mathrm{H}$ 5.48, $\mathrm{N}$ 5.85; found $\mathrm{C} 75.66, \mathrm{H} 5.43, \mathrm{~N} 5.90$.

4.1.3 1-(4-Fluorophenyl)-3-(4-pyridyl)-1,3-propandione (1c)

Yellow powder, yield $72.6 \%$, mp $113-114{ }^{\circ} \mathrm{C} ;{ }^{1} \mathrm{H}$ NMR $\left(\mathrm{CDCl}_{3}, 300\right.$ MHz) d: 4.62 (s, $\left.0.03 \mathrm{H}_{\text {keto }} \mathrm{CH}_{2}\right), 6.84(\mathrm{~s}, 1 \mathrm{H}$, enol CH), 7.20 (t, $2 \mathrm{H}, \mathrm{Ar}-\mathrm{H}, J=$ $8.4 \mathrm{~Hz}), 7.78$ (d, 2H, Py-H, $J=5.7 \mathrm{~Hz}), 8.01-8.06(\mathrm{~m}, 2 \mathrm{H}, \mathrm{Ar}-\mathrm{H}), 8.80(\mathrm{~d}, 2 \mathrm{H}$, Py-H, $J=6.0 \mathrm{~Hz}$ ), 16.52 (br s, $1 \mathrm{H}$, enol OH) ppm; IR (KBr) n: 3438 (w), 3075 (m), $3021(\mathrm{~m}), 1600(\mathrm{~s}), 1545(\mathrm{~m}), 1507(\mathrm{~m}), 1299(\mathrm{~m}), 1227(\mathrm{~s}), 1161(\mathrm{~m}), 844$ (m), $787(\mathrm{~s}) \mathrm{cm}^{-1} ;$ ESI-MS m/z: $244.07[\mathrm{M}+1]^{+}$. Anal. calcd. for $\mathrm{C}_{14} \mathrm{H}_{10} \mathrm{FNO}_{2}: \mathrm{C}$ 69.13, H 4.14, N 5.76; found C 69.27, H 4.11, N 5.79.

4.1.4 1-(4-Methoxyphenyl)-3-(4-pyridyl)-1,3-propandione (1d)

Bright yellow powder, yield $68.4 \%, \mathrm{mp} 121-122{ }^{\circ} \mathrm{C} ;{ }^{1} \mathrm{H} \mathrm{NMR}\left(\mathrm{CDCl}_{3}\right.$, $300 \mathrm{MHz}) \delta: 3.90\left(\mathrm{~s}, 3 \mathrm{H}, \mathrm{OCH}_{3}\right), 4.60\left(\mathrm{~s}, 0.03 \mathrm{H}\right.$ keto $\left.\mathrm{CH}_{2}\right), 6.83(\mathrm{~s}, 1 \mathrm{H}$, enol $\mathrm{CH}), 7.00(\mathrm{~d}, 2 \mathrm{H}, \mathrm{Ar}-\mathrm{H}, J=8.7 \mathrm{~Hz}), 7.78(\mathrm{~d}, 2 \mathrm{H}, \mathrm{Py}-\mathrm{H}, J=6.0 \mathrm{~Hz}), 8.00(\mathrm{~d}$, $2 \mathrm{H}, \mathrm{Ar}-\mathrm{H}, J=9.0 \mathrm{~Hz}), 8.79(\mathrm{~d}, 2 \mathrm{H}, \mathrm{Py}-\mathrm{H}, J=6.0 \mathrm{~Hz}), 16.68(\mathrm{br} \mathrm{s}, 1 \mathrm{H}, \mathrm{enol} \mathrm{OH})$ ppm; IR (KBr) n: 3443 (w), 3031 (m), 2960 (m), 2900 (m), 2840 (w), 1598 (s), $1511(\mathrm{~m}), 1311(\mathrm{~m}), 1260(\mathrm{~s}), 1177(\mathrm{~s}), 1018(\mathrm{~m}), 843(\mathrm{w}), 786(\mathrm{~s}) \mathrm{cm}^{-1}$; ESIMS m/z: $256.21[\mathrm{M}+1]^{+}$. Anal. calcd. for $\mathrm{C}_{15} \mathrm{H}_{13} \mathrm{NO}_{3}$ : C 70.58, H 5.13, N 5.49; found C 70.81, H 5.09, N 5.56.

4.1.5 1-(4-Phenylphenyl)-3-(4-pyridyl)-1,3-propandione (1e)

Yellow powder, yield $70.1 \%$ mp $134-135{ }^{\circ} \mathrm{C} ;{ }^{1} \mathrm{H}$ NMR $\left(\mathrm{CDCl}_{3}, 300\right.$ MHz) $\delta: 4.68\left(\mathrm{~s}, 0.03 \mathrm{H}\right.$ keto $\left.\mathrm{CH}_{2}\right), 6.93(\mathrm{~s}, 1 \mathrm{H}$, enol CH$), 7.40-7.52(\mathrm{~m}, 3 \mathrm{H}$, Ar-H), $7.66(\mathrm{~d}, 2 \mathrm{H}, \mathrm{Ar}-\mathrm{H}, J=7.8 \mathrm{~Hz}), 7.74(\mathrm{~d}, 2 \mathrm{H}, \mathrm{Ar}-\mathrm{H}, J=8.1 \mathrm{~Hz}), 7.82(\mathrm{~d}$, $2 \mathrm{H}, \mathrm{Py}-\mathrm{H}, J=5.1 \mathrm{~Hz}), 8.09(\mathrm{~d}, 2 \mathrm{H}, \mathrm{Ar}-\mathrm{H}, J=8.4 \mathrm{~Hz}), 8.81(\mathrm{~d}, 2 \mathrm{H}, \mathrm{Py}-\mathrm{H}, J=$ $5.4 \mathrm{~Hz}$ ), 16.61 (br s, $1 \mathrm{H}$, enol OH) ppm; IR (KBr) n: 3445 (w), 3041 (m), 1592 (s), $1517(\mathrm{~s}), 1485(\mathrm{~s}), 1409(\mathrm{~m}), 1303(\mathrm{~s}), 1217(\mathrm{~s}), 1058(\mathrm{~m}), 993(\mathrm{~m}), 847$ (s), $768(\mathrm{~s}) \mathrm{cm}^{-1}$; ESI-MS m/z: $302.28[\mathrm{M}+1]^{+}$. Anal. calcd. for $\mathrm{C}_{20} \mathrm{H}_{15} \mathrm{NO}_{2}: \mathrm{C}$ 79.72, H 5.02, N 4.65; found C 79.94, H 4.98, N 4.68.

\subsection{Typical procedure for the synthesis of the $1 \mathrm{H}$-pyrazoles}

Hydrazine hydrate $(6.0 \mathrm{mmol})$ was added dropwise to a solution of the required pyridyl- $\beta$-diketone $(5.0 \mathrm{mmol})$ in $30 \mathrm{~mL}$ absolute ethanol under stirring and the mixture was refluxed at $78^{\circ} \mathrm{C}$ for $3 \mathrm{~h}$. Then the mixture was cooled to room temperature and the solvent was removed by evaporation under reduced pressure to form a precipitate. The precipitate was recrystallized from ethanol (95\%) to obtain the $1 H$-pyrazoles (2a-e).

4.2.1 3-(4-Pyridyl)-5-phenyl-1 $H$-pyrazole (2a)

Colorless powder, yield $88.9 \%, \mathrm{mp} 202-204^{\circ} \mathrm{C}$; IR (KBr): n $3115(\mathrm{~b}, \mathrm{~s})$, $3010(\mathrm{~m}), 1602(\mathrm{~s}), 1487(\mathrm{~m}), 1467(\mathrm{~m}), 1427(\mathrm{~m}), 1391(\mathrm{~m}), 1183(\mathrm{~m}), 1057$ 
$(\mu), 1000(\mathrm{~m}), 957(\mathrm{~m}), 826(\mathrm{~m}), 759(\mathrm{~s}), 708(\mathrm{~s}) \mathrm{cm}^{-1}$; ${ }^{1} \mathrm{H}$ NMR $(300 \mathrm{MHz}$, $\left.\mathrm{CDCl}_{3}\right): \delta 6.96(\mathrm{~s}, 1 \mathrm{H}, \mathrm{C}=\mathrm{CH}), 7.39-7.50(\mathrm{~m}, 3 \mathrm{H}, \mathrm{Ar}-\mathrm{H}), 7.67(\mathrm{~d}, 2 \mathrm{H}, \mathrm{Ar}-\mathrm{H}, J$ $=7.5 \mathrm{~Hz}), 7.72(\mathrm{~d}, 2 \mathrm{H}, \mathrm{Py}-\mathrm{H}, J=4.8 \mathrm{~Hz}), 8.68(\mathrm{~d}, 2 \mathrm{H}, \mathrm{Py}-\mathrm{H}, J=4.5 \mathrm{~Hz}) \mathrm{ppm}$; ESI-MS: m/z 221.16 M+; Anal. calcd. for $\mathrm{C}_{14} \mathrm{H}_{11} \mathrm{~N}_{3}$ : C 76.00; H 5.01; N 18.99; found C 76.24; H 4.97; N 19.03.

4.2.2 3-(4-Pyridyl)-5-(4-methylphenyl)-1 $H$-pyrazole (2b)

Colorless powder, yield $91.3 \%$, mp $210-211^{\circ} \mathrm{C}$; IR (KBr): n $3116(\mathrm{~m})$, $3071(\mathrm{~m}), 3006(\mathrm{~m}), 2920(\mathrm{w}), 2855(\mathrm{w}), 1604(\mathrm{~s}), 1510(\mathrm{~m}), 1461(\mathrm{~m}), 1185$ (m), 1063 (m), 965 (m), 865 (w), $822(\mathrm{~s}) \mathrm{cm}^{-1} ;{ }^{1} \mathrm{H}$ NMR (300 MHz, $\left.\mathrm{CDCl}_{3}\right): \delta$ $2.38\left(\mathrm{~s}, 3 \mathrm{H}, \mathrm{CH}_{3}\right), 6.89(\mathrm{~s}, 1 \mathrm{H}, \mathrm{C}=\mathrm{CH}), 7.21(\mathrm{~d}, 2 \mathrm{H}, \mathrm{Ar}-\mathrm{H}, J=7.8 \mathrm{~Hz}), 7.53(\mathrm{~d}$, $2 \mathrm{H}, \mathrm{Ar}-\mathrm{H}, J=7.8 \mathrm{~Hz}), 7.66$ (d, 2H, Py-H, $J=4.8 \mathrm{~Hz}), 8.63$ (d, 2H, Py-H, $J=$ 5.1 Hz) ppm; ESI-MS: m/z 235.07 M+; Anal. calcd. for $\mathrm{C}_{15} \mathrm{H}_{13} \mathrm{~N}_{3}: \mathrm{C} 76.57 ; \mathrm{H}$ 5.57; N 17.86; found C 76.80; H 5.52; N 17.89 .

4.2.3 3-(4-Pyridyl)-5-(4-fluorophenyl)-1 $H$-pyrazole (2c)

Colorless powder, yield $92.9 \%$, mp $189-191^{\circ} \mathrm{C}$; IR (KBr): n 3135 (b, s), $3048(\mathrm{~m}), 1607$ (s), $1507(\mathrm{~s}), 1458(\mathrm{~m}), 1232(\mathrm{~s}), 1168(\mathrm{~m}), 1099(\mathrm{~m}), 1064$ (m), $973(\mathrm{~m}), 836(\mathrm{~s}), 800(\mathrm{~s}), 698(\mathrm{~m}) \mathrm{cm}^{-1} ;{ }^{1} \mathrm{H}$ NMR (300 MHz, CDCl $): \delta$ $6.90(\mathrm{~s}, 1 \mathrm{H}, \mathrm{C}=\mathrm{CH}), 7.13(\mathrm{t}, 2 \mathrm{H}, \mathrm{Ar}-\mathrm{H}, J=8.1 \mathrm{~Hz}), 7.64(\mathrm{~d}, 2 \mathrm{H}, \mathrm{Ar}-\mathrm{H}, J=8.7$ $\mathrm{Hz}), 7.67$ (d, 2H, Py-H, $J=5.1 \mathrm{~Hz}), 8.66(\mathrm{~d}, 2 \mathrm{H}, \mathrm{Py}-\mathrm{H}, J=4.8 \mathrm{~Hz}) \mathrm{ppm}$; ESIMS: $\mathrm{m} / \mathrm{z} 239.14 \mathrm{M}^{+}$; Anal. calcd. for $\mathrm{C}_{14} \mathrm{H}_{10} \mathrm{FN}_{3}$ : C 70.28; $\mathrm{H}$ 4.21; $\mathrm{N}$ 17.56; found C 70.50; H 4.18; N 17.60.

4.2.4 3-(4-Pyridyl)-5-(4-methoxyphenyl)- $1 H$-pyrazole (2d)

Colorless needles, yield 90.5\%, mp 196-197 ${ }^{\circ} \mathrm{C}$; IR (KBr): n 3117 (m), $3072(\mathrm{~m}), 3026(\mathrm{~m}), 2915(\mathrm{w}), 2845$ (w), 1607 (s), $1509(\mathrm{~s}), 1458(\mathrm{~m}), 1290$ (m), $1252(\mathrm{~s}), 1180(\mathrm{~s}), 1029(\mathrm{~m}), 964(\mathrm{~m}), 828(\mathrm{~s}), 793(\mathrm{~m}), 697(\mathrm{~m}) \mathrm{cm}^{-1} ;{ }^{1} \mathrm{H}$ NMR (300 MHz, CDCl $): \delta 3.85\left(\mathrm{~s}, 3 \mathrm{H}, \mathrm{OCH}_{3}\right), 6.85(\mathrm{~s}, 1 \mathrm{H}, \mathrm{C}=\mathrm{CH}), 6.96(\mathrm{~d}$, $2 \mathrm{H}, \mathrm{Ar}-\mathrm{H}, J=7.8 \mathrm{~Hz}$ ), 7.58 (d, 2H, Ar-H, $J=8.1 \mathrm{~Hz}$ ), 7.69 (d, 2H, Py-H, $J=$ $3.3 \mathrm{~Hz}), 8.65(\mathrm{~d}, 2 \mathrm{H}$, Py-H, $J=2.1 \mathrm{~Hz}) \mathrm{ppm}$; ESI-MS: m/z $251.10 \mathrm{M}^{+}$; Anal. calcd. for $\mathrm{C}_{15} \mathrm{H}_{13} \mathrm{~N}_{3} \mathrm{O}$ : C 71.70; $\mathrm{H} \mathrm{5.21;} \mathrm{N} \mathrm{16.72;} \mathrm{found} \mathrm{C} 71.92 ; \mathrm{H} 5.17 ; \mathrm{N}$ 16.74 .

4.2.5 3-(4-Pyridyl)-5-(4-phenylphenyl)-1H-pyrazole (2e)

Colorless powder, yield $92.8 \%$, mp 267-269 ${ }^{\circ} \mathrm{C}$; IR (KBr): n $3133(\mathrm{~m})$, $3031(\mathrm{~m}), 1603(\mathrm{~s}), 1480(\mathrm{~m}), 1424(\mathrm{~m}), 1351(\mathrm{~m}), 1226(\mathrm{~m}), 1181(\mathrm{~m}), 1058$ (m), 1000 (m), 955 (m), 837 (s), 762 (s), 693 (m) cm ${ }^{-1}$; ${ }^{1} \mathrm{H}$ NMR (300 MHz, $\left.\mathrm{CDCl}_{3}\right): \delta 7.00(\mathrm{~s}, 1 \mathrm{H}, \mathrm{C}=\mathrm{CH}), 7.40-7.42(\mathrm{~m}, 3 \mathrm{H}, \mathrm{Ar}-\mathrm{H}), 7.47(\mathrm{~d}, 2 \mathrm{H}, \mathrm{Ar}-\mathrm{H}$, $J=7.2 \mathrm{~Hz}), 7.50(\mathrm{~d}, 2 \mathrm{H}, \mathrm{Ar}-\mathrm{H}, J=6.9 \mathrm{~Hz}), 7.65(\mathrm{~d}, 2 \mathrm{H}, \mathrm{Ar}-\mathrm{H}, J=7.8 \mathrm{~Hz})$, $7.72(\mathrm{~d}, 2 \mathrm{H}, \mathrm{Py}-\mathrm{H}, J=6.3 \mathrm{~Hz}), 8.69(\mathrm{~d}, 2 \mathrm{H}, \mathrm{Py}-\mathrm{H}, J=5.1 \mathrm{~Hz}) \mathrm{ppm}$; ESI-MS: $\mathrm{m} / \mathrm{z} 297.11 \mathrm{M}^{+}$; Anal. calcd. for $\mathrm{C}_{20} \mathrm{H}_{15} \mathrm{~N}_{3}$ : C 80.78; H 5.08; N 14.13; found $\mathrm{C}$ 80.99; H 5.04; N 14.18.

4.3 Typical procedure for the synthesis of the1-acetyl-pyrazoles

Acetyl chloride $(4.0 \mathrm{mmol})$ was added dropwise to a mixture of the corresponding $1 \mathrm{H}$-pyrazoles $(2.0 \mathrm{mmol})$ and triethylamine $(4.0 \mathrm{mmol})$ in 20 $\mathrm{mL}$ THF. The mixture was refluxed for $5 \mathrm{~h}$ until the precipitate was formed. The precipitate was filtered and the filtrate was concentrated to obtain a solid product. The solid was recrystallized from ethanol-cyclohexane (1:1) to give the 1-acetyl pyrazoles (3a-e).

4.3.1 1-Acetyl-3-(4-pyridyl)-5-phenylpyrazole (3a)

Yellow powder, yield $87.2 \%$, mp $129-131^{\circ} \mathrm{C}$; IR (KBr): $\mathrm{n} 3089(\mathrm{~m}), 3005$ (m), $2936(\mathrm{w}), 2855(\mathrm{w}), 1740(\mathrm{~s}), 1620(\mathrm{~m}), 1557(\mathrm{~m}), 1491(\mathrm{~m}), 1434(\mathrm{~m})$, $1368(\mathrm{~m}), 1299(\mathrm{~s}), 1219(\mathrm{~m}), 1105(\mathrm{~m}), 1026(\mathrm{~m}), 945(\mathrm{~s}), 831(\mathrm{~m}), 761(\mathrm{~s})$, $690(\mathrm{~s}) \mathrm{cm}^{-1} ;{ }^{1} \mathrm{H}$ NMR $\left(300 \mathrm{MHz}, \mathrm{CDCl}_{3}\right): \delta 2.83\left(\mathrm{~s}, 3 \mathrm{H}, \mathrm{CH}_{3}\right), 6.85(\mathrm{~s}, 1 \mathrm{H}$, $\mathrm{C}=\mathrm{CH}), 7.42-7.52(\mathrm{~m}, 5 \mathrm{H}, \mathrm{Ar}-\mathrm{H}), 8.02(\mathrm{~d}, 2 \mathrm{H}, \mathrm{Py}-\mathrm{H}, J=6.0 \mathrm{~Hz}), 8.76(\mathrm{~d}$, $2 \mathrm{H}, \mathrm{Py}-\mathrm{H}, J=6.0 \mathrm{~Hz}$ ) ppm; ESI-MS: m/z $263.75[\mathrm{M}+1]^{+}$; Anal. calcd. for $\mathrm{C}_{16} \mathrm{H}_{13} \mathrm{~N}_{3} \mathrm{O}$ : C 72.99; $\mathrm{H} 4.98 ; \mathrm{N}$ 15.96; found $\mathrm{C} 73.26 ; \mathrm{H} 4.94 ; \mathrm{N} 15.99$.

4.3.2 1-Acetyl-3-(4-pyridyl)-5-(4-methylphenyl) pyrazole (3b)

Yellow powder, yield $86.6 \%$, mp $138-139^{\circ} \mathrm{C}$; IR (KBr): $\mathrm{n} 3085(\mathrm{~m}), 3024$ (m), $2922(\mathrm{w}), 2860(\mathrm{w}), 1731(\mathrm{~s}), 1673(\mathrm{~m}), 1614(\mathrm{~m}), 1561(\mathrm{w}), 1494(\mathrm{w})$, $1421(\mathrm{~m}), 1370(\mathrm{~m}), 1331(\mathrm{~s}), 1230(\mathrm{~m}), 946(\mathrm{~m}), 816(\mathrm{~s}) \mathrm{cm}^{-1} ;{ }^{1} \mathrm{H}$ NMR $(300$ $\left.\mathrm{MHz}, \mathrm{CDCl}_{3}\right): \delta 2.42\left(\mathrm{~s}, 3 \mathrm{H}, \mathrm{CH}_{3}\right), 2.81\left(\mathrm{~s}, 3 \mathrm{H}, \mathrm{CH}_{3}\right), 6.78(\mathrm{~s}, 1 \mathrm{H}, \mathrm{C}=\mathrm{CH})$, 7.29 (d, $2 \mathrm{H}, \mathrm{Ar}-\mathrm{H}, J=8.7 \mathrm{~Hz}), 7.39$ (d, 2H, Py-H, $J=6.0 \mathrm{~Hz}), 7.77-7.80(\mathrm{~m}$, $2 \mathrm{H}, \mathrm{Ar}-\mathrm{H}), 8.68$ (d, 2H, Py-H, $J=6.0 \mathrm{~Hz}) \mathrm{ppm}$; ESI-MS: m/z $277.84[\mathrm{M}+1]^{+}$; Anal. calcd. for $\mathrm{C}_{17} \mathrm{H}_{15} \mathrm{~N}_{3} \mathrm{O}$ : C 73.63; H 5.45; N 15.15; found C 73.85; H 5.41; N 15.18 .

4.3.3 1-Acetyl-3-(4-pyridyl)-5-(4-fluorophenyl) pyrazole (3c)

Bright yellow powder, yield $84.8 \%$, mp $142-143{ }^{\circ} \mathrm{C}$; IR (KBr): n 3100 (m), $3010(\mathrm{~m}), 2960(\mathrm{w}), 2870(\mathrm{w}), 1739(\mathrm{~s}), 1608(\mathrm{~m}), 1506(\mathrm{~m}), 1425(\mathrm{~m})$, $1371(\mathrm{~m}), 1321(\mathrm{~s}), 1225$ (s), $1160(\mathrm{~m}), 1107$ (m), 947 (m), $817(\mathrm{~s}) \mathrm{cm}^{-1} ;{ }^{1} \mathrm{H}$ NMR (300 MHz, $\left.\mathrm{CDCl}_{3}\right): \delta 2.81\left(\mathrm{~s}, 3 \mathrm{H}, \mathrm{CH}_{3}\right), 6.79(\mathrm{~s}, 1 \mathrm{H}, \mathrm{C}=\mathrm{CH}), 7.09-7.19$ (m, 2H, Ar-H), 7.41-7.45 (m, 2H, Ar-H), 7.87 (d, 2H, Py-H, $J=5.1 \mathrm{~Hz}), 8.69$ (d, $2 \mathrm{H}, \mathrm{Py}-\mathrm{H}, J=5.1 \mathrm{~Hz}$ ) ppm; ESI-MS: m/z $281.88[\mathrm{M}+1]^{+}$; Anal. calcd. for $\mathrm{C}_{16} \mathrm{H}_{12} \mathrm{FN}_{3} \mathrm{O}$ : C 68.32; $\mathrm{H} 4.30 ; \mathrm{N} \mathrm{14.94;} \mathrm{found} \mathrm{C} 68.56 ; \mathrm{H} 4.27$; 14.98.

4.3.4 1-Acetyl-3-(4-pyridyl)-5-(4-methoxyphenyl) pyrazole (3d)

Yellow powder, yield $83.6 \%$, mp $158-159^{\circ} \mathrm{C}$; IR (KBr): $\mathrm{n} 3106(\mathrm{~m}), 3000$ (w), $2985(\mathrm{w}), 2840(\mathrm{w}), 1735(\mathrm{~s}), 1670(\mathrm{~m}), 1610(\mathrm{~m}), 1505(\mathrm{~m}), 1427(\mathrm{~m})$, $1367(\mathrm{~m}), 1300(\mathrm{~s}), 1250(\mathrm{~s}), 1180(\mathrm{~m}), 1109(\mathrm{~m}), 1029(\mathrm{~s}), 945(\mathrm{~s}), 810(\mathrm{~s})$ $\mathrm{cm}^{-1}$; ${ }^{1} \mathrm{H}$ NMR $\left(300 \mathrm{MHz}, \mathrm{CDCl}_{3}\right): \delta 2.81\left(\mathrm{~s}, 3 \mathrm{H}, \mathrm{CH}_{3}\right), 3.86\left(\mathrm{~s}, 3 \mathrm{H}, \mathrm{CH}_{3}\right)$, $6.73(\mathrm{~s}, 1 \mathrm{H}, \mathrm{C}=\mathrm{CH}), 6.94-7.01(\mathrm{~m}, 2 \mathrm{H}, \mathrm{Ar}-\mathrm{H}), 7.39(\mathrm{~d}, 2 \mathrm{H}, \mathrm{Ar}-\mathrm{H}, J=7.8 \mathrm{~Hz})$, 7.76-7.84 (m, 2H, Py-H), 8.68-8.71 (m, 2H, Py-H) ppm; ESI-MS: m/z 293.85 $[\mathrm{M}+1]^{+}$; Anal. calcd. for $\mathrm{C}_{17} \mathrm{H}_{15} \mathrm{~N}_{3} \mathrm{O}_{2}: \mathrm{C} 69.61 ; \mathrm{H} 5.15 ; \mathrm{N}$ 14.33; found $\mathrm{C} 69.89$; H $5.11 ; \mathrm{N} 14.37$.

4.3.5 1-Acetyl-3-(4-pyridyl)-5-(4-phenylphenyl) pyrazole (3e)

Yellow crystals, yield $85.9 \%$, mp 166-168 ${ }^{\circ} \mathrm{C}$; IR (KBr): n 3090 (w), 3035 (w), $2920(\mathrm{w}), 2860(\mathrm{w}), 1740(\mathrm{~s}), 1609(\mathrm{~m}), 1559(\mathrm{~m}), 1485(\mathrm{~m}), 1421(\mathrm{~m})$ $1368(\mathrm{~m}), 1295(\mathrm{~s}), 1216(\mathrm{~m}), 1108(\mathrm{~m}), 1017(\mathrm{~m}), 946(\mathrm{~m}), 832(\mathrm{~s}) \mathrm{cm}^{-1} ;{ }^{1} \mathrm{H}$ $\operatorname{NMR}\left(300 \mathrm{MHz}, \mathrm{CDCl}_{3}\right): \delta 2.84\left(\mathrm{~s}, 3 \mathrm{H}, \mathrm{CH}_{3}\right), 6.83(\mathrm{~s}, 1 \mathrm{H}, \mathrm{C}=\mathrm{CH}), 7.38-7.56$ (m, 5H, Ar-H), 7.63-7.72 (m, 4H, Ar-H), 7.82-7.98 (m, 2H, Py-H), 8.68-8.74 (m, 2H, Py-H) ppm; ESI-MS: m/z $339.89[\mathrm{M}+1]^{+}$; Anal. calcd. for $\mathrm{C}_{22} \mathrm{H}_{17} \mathrm{~N}_{3} \mathrm{O}$ : C 77.86; H 5.05; N 12.38; found C 78.11; H 5.01; N 12.42 .

4.4 Typical procedure for the synthesis of the1-benzoyl-pyrazoles

Same procedure as for the 1-acetyl-pyrazoles, but the reaction mixture with the required $1 \mathrm{H}$-pyrazoles $(2.0 \mathrm{mmol})$, triethylamine $(4.0 \mathrm{mmol})$ and benzoyl chloride ( $4.0 \mathrm{mmol})$. The 1-benzoyl pyrazoles (4a-e) were obtained.

4.4.11-Benzoyl-3-(4-pyridyl)-5-phenylpyrazole (4a)

Yellow powder, yield $87.6 \%$, mp 111-113 ${ }^{\circ} \mathrm{C}$; IR (KBr): n $3066(\mathrm{~m}), 1710$ $(\mathrm{s}), 1633(\mathrm{~s}), 1521(\mathrm{~m}), 1488(\mathrm{~m}), 1446(\mathrm{~m}), 1405(\mathrm{~m}), 1295(\mathrm{~s}), 1241(\mathrm{~m})$ $1193(\mathrm{~m}), 1076(\mathrm{~m}), 903(\mathrm{~s}), 818(\mathrm{~s}), 766(\mathrm{~s}) \mathrm{cm}^{-1} ;{ }^{1} \mathrm{H} \mathrm{NMR}\left(300 \mathrm{MHz}, \mathrm{CDCl}_{3}\right)$ : $\delta 7.13(\mathrm{~s}, 1 \mathrm{H}, \mathrm{C}=\mathrm{CH}), 7.47-7.59(\mathrm{~m}, 3 \mathrm{H}, \mathrm{Ar}-\mathrm{H}), 7.70-7.75(\mathrm{~m}, 2 \mathrm{H}, \mathrm{Ar}-\mathrm{H})$, 8.02-8.05 (m, 3H, Ar-H), 8.18 (d, 2H, Ar-H, $J=7.2 \mathrm{~Hz}), 8.35$ (d, 2H, Py-H, $J$ $=6.6 \mathrm{~Hz}), 8.85(\mathrm{~d}, 2 \mathrm{H}$, Py-H, $J=5.7 \mathrm{~Hz})$ ppm; ESI-MS: m/z $326.03[\mathrm{M}+1]^{+}$; Anal. calcd. for $\mathrm{C}_{21} \mathrm{H}_{15} \mathrm{~N}_{3} \mathrm{O}$ : C 77.52; $\mathrm{H} 4.65 ; \mathrm{N}$ 12.91; found $\mathrm{C}$ 77.80; $\mathrm{H}$ 4.61; N 12.95 .

4.4.2 1-Benzoyl-3-(4-pyridyl)-5-(4-methylphenyl) pyrazole (4b)

Yellow powder, yield $82.6 \%$, mp 130-131 ${ }^{\circ} \mathrm{C}$; IR (KBr): n $3079(\mathrm{~m}), 2940$ (w), $2885(\mathrm{w}), 1713(\mathrm{~s}), 1633(\mathrm{~s}), 1497(\mathrm{~m}), 1435(\mathrm{~m}), 1317(\mathrm{~s}), 1185(\mathrm{~m}), 900$ (s), $820(\mathrm{~s}), 719(\mathrm{~m}) \mathrm{cm}^{-1} ;{ }^{1} \mathrm{H}$ NMR $\left(300 \mathrm{MHz}, \mathrm{CDCl}_{3}\right): \delta 2.41\left(\mathrm{~s}, 3 \mathrm{H}, \mathrm{CH}_{3}\right)$, $7.11(\mathrm{~s}, 1 \mathrm{H}, \mathrm{C}=\mathrm{CH}), 7.37(\mathrm{~d}, 2 \mathrm{H}, \mathrm{Ar}-\mathrm{H}, J=8.1 \mathrm{~Hz}), 7.53-7.60(\mathrm{~m}, 2 \mathrm{H}, \mathrm{Ar}-\mathrm{H})$, 7.70-7.77 (m, 3H, Ar-H), 8.04 (d, 2H, Ar-H, $J=7.2 \mathrm{~Hz}$ ), 8.34 (d, 2H, Py-H, $J$ $=4.5 \mathrm{~Hz}), 8.84(\mathrm{~d}, 2 \mathrm{H}, \mathrm{Py}-\mathrm{H}, J=5.4 \mathrm{~Hz}) \mathrm{ppm}$; ESI-MS: m/z $340.25[\mathrm{M}+1]^{+}$; Anal. calcd. for $\mathrm{C}_{22} \mathrm{H}_{17} \mathrm{~N}_{3} \mathrm{O}$ : C 77.86; H 5.05; N 12.38; found C 78.13; H 5.01; N 12.41 .

\subsubsection{1-Benzoyl-3-(4-pyridyl)-5-(4-fluorophenyl) pyrazole (4c)}

Yellow powder, yield $85.2 \%$, mp $128-139^{\circ} \mathrm{C}$; IR (KBr): n $3058(\mathrm{~m}), 1721$ (s), $1634(\mathrm{~s}), 1601(\mathrm{~m}), 1491(\mathrm{~s}), 1434(\mathrm{~m}), 1318(\mathrm{~s}), 1232(\mathrm{~m}), 1166(\mathrm{~m}), 1099$ (m), $906(\mathrm{~m}), 818(\mathrm{~s}), 719(\mathrm{~m}) \mathrm{cm}^{-1} ;{ }^{1} \mathrm{H}$ NMR $\left(300 \mathrm{MHz}, \mathrm{CDCl}_{3}\right): \delta 7.09(\mathrm{~s}$, $1 \mathrm{H}, \mathrm{C}=\mathrm{CH}), 7.17(\mathrm{t}, 2 \mathrm{H}, \mathrm{Ar}-\mathrm{H}, J=8.4 \mathrm{~Hz}), 7.45-7.50(\mathrm{~m}, 2 \mathrm{H}, \mathrm{Ar}-\mathrm{H}), 7.54(\mathrm{t}$, $2 \mathrm{H}, \mathrm{Ar}-\mathrm{H}, J=7.8 \mathrm{~Hz}), 7.71-7.76(\mathrm{~m}, 1 \mathrm{H}, \mathrm{Ar}-\mathrm{H}), 8.02-8.10$ (m, $2 \mathrm{H}, \mathrm{Ar}-\mathrm{H}), 8.31$ $(\mathrm{d}, 2 \mathrm{H}, \mathrm{Py}-\mathrm{H}, J=6.0 \mathrm{~Hz}), 8.80(\mathrm{~d}, 2 \mathrm{H}, \mathrm{Py}-\mathrm{H}, J=6.0 \mathrm{~Hz}) \mathrm{ppm} ;$ ESI-MS: $\mathrm{m} / \mathrm{z}$ $344.00[\mathrm{M}+1]^{+}$; Anal. calcd. for $\mathrm{C}_{21} \mathrm{H}_{14} \mathrm{FN}_{3} \mathrm{O}$ : C 73.46; $\mathrm{H} 4.11 ; \mathrm{N} \mathrm{12.24}$; found C 73.69; H 4.08; N 12.28

4.4.4 1-Benzoyl-3-(4-pyridyl)-5-(4-methoxyphenyl) pyrazole (4d)

Yellow powder, yield 80.0\%, mp 96-97 ${ }^{\circ} \mathrm{C}$; IR $(\mathrm{KBr}): \mathrm{n} 3086(\mathrm{~m}), 2994$ (m), $2830(\mathrm{~m}), 1717(\mathrm{~s}), 1633(\mathrm{~s}), 1497(\mathrm{~s}), 1422(\mathrm{~m}), 1322(\mathrm{~s}), 1293(\mathrm{~s}), 1255$ (s), $1184(\mathrm{~s}), 1107$ (m), 1031 (m), 953 (m), 902 (s), $814(\mathrm{~s}), 715(\mathrm{~m}) \mathrm{cm}^{-1} ;{ }^{1} \mathrm{H}$ NMR $\left(300 \mathrm{MHz}, \mathrm{CDCl}_{3}\right): \delta 3.85\left(\mathrm{~s}, 3 \mathrm{H}, \mathrm{OCH}_{3}\right), 6.94(\mathrm{~d}, 2 \mathrm{H}, \mathrm{Ar}-\mathrm{H}, J=6.9$ $\mathrm{Hz}), 7.11(\mathrm{~s}, 1 \mathrm{H}, \mathrm{C}=\mathrm{CH}), 7.41(\mathrm{~d}, 2 \mathrm{H}, \mathrm{Ar}-\mathrm{H}, J=8.7 \mathrm{~Hz}), 7.55(\mathrm{t}, 2 \mathrm{H}, \mathrm{Ar}-\mathrm{H}, J=$ $7.5 \mathrm{~Hz}), 7.68-7.74(\mathrm{~m}, 1 \mathrm{H}, \mathrm{Ar}-\mathrm{H}), 8.02(\mathrm{~d}, 2 \mathrm{H}, \mathrm{Ar}-\mathrm{H}, J=7.2 \mathrm{~Hz}), 8.34(\mathrm{~d}, 2 \mathrm{H}$, Py-H, $J=6.6 \mathrm{~Hz}$ ), 8.90 (d, 2H, Py-H, $J=6.3 \mathrm{~Hz}) \mathrm{ppm}$; ESI-MS: m/z 356.05 $[\mathrm{M}+1]^{+}$; Anal. calcd. for $\mathrm{C}_{22} \mathrm{H}_{17} \mathrm{~N}_{3} \mathrm{O}_{2}$ : C 74.35; $\mathrm{H} 4.82 ; \mathrm{N} 11.82$; found $\mathrm{C} 74.62$; H 4.78; N 11.86 .

4.4.5 1-Benzoyl-3-(4-pyridyl)-5-(4-phenylphenyl) pyrazole (4e)

Colorless powder, yield $84.3 \%$, mp $136-138^{\circ} \mathrm{C}$; IR (KBr): n $3062(\mathrm{~m})$, 3012 (m), 1711 (s), 1632 (s), 1603 (m), $1516(\mathrm{~m}), 1488$ (m), 1447 (m), 1409 $(\mathrm{m}), 1295(\mathrm{~s}), 1200(\mathrm{~s}), 949(\mathrm{~m}), 902(\mathrm{~s}), 830(\mathrm{~s}), 767(\mathrm{~m}), 716(\mathrm{~s}) \mathrm{cm}^{-1} ;{ }^{1} \mathrm{H}$ NMR (300 MHz, $\mathrm{CDCl}_{3}$ ): d 7.19 (s, 1H, C=CH), 7.36-7.49 (m, 3H, Ar-H), 7.54-7.76 (m, 9H), $8.07(\delta, 2 \mathrm{H}, \mathrm{Ar}-\mathrm{H}, J=6.9 \mathrm{~Hz}), 8.35(\mathrm{~d}, 2 \mathrm{H}, \mathrm{Py}-\mathrm{H}, J=6.3$ $\mathrm{Hz}$ ), 8.87 (d, 2H, Py-H, $J=6.3 \mathrm{~Hz}$ ) ppm; ESI-MS: m/z 402.39 [M+1] $]^{+}$; Anal. calcd. for $\mathrm{C}_{27} \mathrm{H}_{19} \mathrm{~N}_{3} \mathrm{O}$ : C 80.78; H 4.77; N 10.47; found $\mathrm{C} 80.94 ; \mathrm{H} 4.73$; N 10.50 .

\subsection{Procedure for anti-microbial activity}

The synthesized compounds (3a-e and $\mathbf{4 a - e}$ ) were screened for their in vitro antibacterial activity against Escherichia coli and Staphylococcus aureus and antifungal activity against Pyricularia oryzae and Rhizoctnia solani. The anti-microbial activity was performed by disc diffusion method $[16,17]$ at a concentration of $50 \mathrm{mg} \mathrm{L}^{-1}$. Muller-Hinton agar (Hi-Media) was employed as culture medium and DMSO was used as solvent control for anti-microbial activity. Norfloxacin and Triadimefon were used as standard for antibacterial 
and antifungal activities, respectively. The inhibition zones were measured in $\mathrm{mm}$ at the end of an incubation period of $24 \mathrm{~h}$ at $37^{\circ} \mathrm{C}$ for bacteria and $72 \mathrm{~h}$ at $24{ }^{\circ} \mathrm{C}$ for fungi.

\section{SUPPLEMENTARY MATERIAL}

Crystallographic data have been deposited with the Cambridge Crystallographic Data Center. Supplementary publication No. CCDC 965134. Copies of data may be obtained free of charge from the Director, CCDC, 12 Union Road, Cambridge, CB2 1EZ, UK (Fax: +44-1223-336033; email: deposit@.ccdc. cam.ac.uk).

\section{ACKNOWLEDGMENT}

Financial support for this work from National Natural Science Foundation of China (no. 21273065).

\section{REFERENCES}

1. M. Rahimizadeh, M. Pordel, M. Bakavoli, S. Rezaeian, A. Sadeghian, World J. Microbiol. Biotechnol. 26, 317, (2010).

2. C. S. Reddy, M. V. Devi, G. R. Kumar, L. S. Rao, A. Nagaraj, Indian J. Chem. 50B, 1181, (2011).

3. M. Amir, S. Kumar, Indian J. Chem. 44B, 2532, (2005).

4. C. Almansa, L. A. Gómez, F. L. Cavaleanti, A. F. de Arriba, J. GarcíaRafanell, J. Forn, J. Med. Chem. 40, 547, (1997).
5. D. M. Bailey, P. E. Hansen, A. G. Hlavac, E. R. Baizman, J. Pearl, A. F. DeFelice, M. E. Feigenson, J. Med. Chem. 28, 256, (1985).

6. A. S. Tantawy, M. N. A. Nasr, M. A. A. El-Sayed, S. S. Tawfik, Med. Chem. Res. 21, 4139, (2012).

7. H. Katayama, T. Oshiyama, Can. J. Chem. 75, 913, (1997).

8. R. Kalirajan, L. Rathore, S. Jubie, B. Gowramma, S. Gomathy, S. Sankar, Indian J. Chem. 50B, 1794, (2011).

9. E. Banoğlu, M. Şüküroğlu, B.Ç. Ergün, S.N. Baytaş, E. Aypar, M. Ark, Turk. J. Chem. 31, 677, (2007).

10. A.V. Gadakh, C. Pandit, S. S. Rindhe, B.K. Karale, Bioorg. Med. Chem. Lett. 20, 5572, (2010).

11. W.-L. Dong, J.-Y. Xu, L.-X. Xiong, X.-H. Liu, Z.-M. Li, Chin. J. Chem. 27, 579, (2009).

12. F.A. Bassyouni, H.A. Tawfik, A.M. Soliman, M.A. Rehim, Res. Chem. Intermed. 38, 1291, (2012).

13. M.A. El-borai, H.F. Rizk, M.F. Abd-Aal, I.Y. El-Deeb, Eur. J. Med. Chem. 48, 92, (2012).

14. B. A. Bhat, S. C. Puri, Synth. Commun. 35, 1135, (2005).

15. F. Chimenti, A. Bolasco, F. Manna, D. Secci, P. Chimenti, A. Granese, O. Befani, P. Turini, S. Alcaro, F. Ortuso, Chem. Biol. Drug Des. 67, 206, (2006).

16. T. Premkumar, S. Govindarajan, World J. Microbiol. Biotechnol. 21, 479, (2005).

17. W. R. Kirkpatrick, T. M. Turner, A. W. Fothergill, D. I. Mccarthy, S. W. Redding, M. G. Rinaldi, T. F. Patterson, J. Clin. Microbiol. 36, 3429, (1998). 